\title{
Entrelacs
}

ENTRELACS Cinéma et audiovisuel

$10 \mid 2013$

Le Toucher

\section{Comprendre par les sens : Pour une approche tactile de L'Apollonide}

\section{Romain Chareyron}

\section{(2) OpenEdition}

Journals

Édition électronique

URL : http://journals.openedition.org/entrelacs/528

DOI : $10.4000 /$ entrelacs.528

ISSN : 2261-5482

Éditeur

Éditions Téraèdre

Référence électronique

Romain Chareyron, «Comprendre par les sens : Pour une approche tactile de L'Apollonide », Entrelacs [En ligne], 10 | 2013, mis en ligne le 12 septembre 2013, consulté le 21 avril 2019. URL : http:// journals.openedition.org/entrelacs/528; DOI : 10.4000/entrelacs.528

Ce document a été généré automatiquement le 21 avril 2019

Tous droits réservés 


\title{
Comprendre par les sens: Pour une approche tactile de L'Apollonide
}

\author{
Romain Chareyron
}

1 La peau et le toucher jouent un rôle fondateur dans l'économie visuelle du film de Bertrand Bonello, L'Apollonide (2011). En plaçant sa caméra au cœur d'une maison close de luxe «[...] où les filles habillées comme des princesses attendent le client dans des chambres tendues de velours noir $"^{1}$, le film se veut une expérience éminemment sensuelle, nous entraînant dans un univers tout entier dédié à la volupté et aux plaisirs des sens. Le récit souhaitant également fournir une retranscription réaliste et détaillée du quotidien d'une maison de tolérance, le toucher revêt des formes diverses, allant de la caresse sensuelle des clients aux gestes d'affection et de solidarité que les prostituées ont les unes pour les autres. Comme le film n'élude pas non plus le versant mortifère et sombre du métier, le toucher se matérialise aussi sous une forme plus violente, en soulignant l'impuissance des femmes face à la brutalité et au sadisme des hommes.

\section{Les implications esthétiques et narratives du toucher dans L'Apollonide}

2 C'est sur la force affective contenue dans cette interaction entre les corps que nous souhaitons porter notre attention, afin d'observer comment les différents types de toucher mis en place par le récit participent à notre compréhension du féminin tel que l'envisage le film. Nous observerons également comment ces différentes formes de toucher en appellent à des procédés de mise en scène variés, dont le but est de communiquer aux spectateurs la complexité de la condition féminine au sein de la maison close. En mettant en place divers régimes visuels, le film va chercher à modifier la nature du lien qu'il noue avec celui qui perçoit, ce dernier passant de la position de simple voyant, maintenu à distance par la représentation, à celle de participant actif, engagé physiquement et émotionnellement dans la matière vivante des images. 
3 Dans son ouvrage, intitulé The Cultural Politics of Emotions, Sara Ahmed propose de comprendre les émotions comme "des pratiques sociales et culturelles ${ }^{2}$, entendant par là qu'il nous faut repenser les émotions, non plus comme correspondant à l'extériorisation d'un ressenti individuel, mais comme naissant du contact entre les corps, ainsi que de la forme que prend ce contact. Ahmed considère l'émotion comme une force mouvante qui circule entre les corps et informe notre rapport aux autres, et non comme une manifestation prédéterminée qui viendrait s'imprimer sur nos comportements :

Les émotions ne sont pas quelque chose d'inhérent au "je" ou au "nous". C'est à travers les émotions, ou la manière dont nous interagissons avec les objets ou les personnes, que des surfaces et des frontières se mettent en place : le "je" ou le "nous" est façonné par son contact avec les autres, jusqu'à en prendre parfois la forme. ${ }^{3}$

4 C'est en suivant le modèle mis en place par Ahmed, et en déplaçant l'objet de notre attention des corps aux modalités présidant à leur mise en contact, que nous avons choisi d'aborder la symbolique du toucher dans le film de Bonello. La progression de notre analyse suivra les différentes formes que recouvre le toucher au sein du film, commençant à la surface de la peau, pour s'intéresser à la symbolique de la caresse, puis s'aventurant plus en profondeur pour discuter du toucher comme geste menant à la blessure qui vient entailler les chairs. Nous verrons ainsi comment les différentes formes de contact mises en scène par le film donnent lieu à des ressentis de nature diverse, tant chez les personnages qu'au niveau spectatoriel.

\section{Les ambiguïtés du toucher}

\section{Le toucher comme marque de la réification du féminin}

5 Le toucher tel que manifesté par la caresse opère sur un mode duel, en ce qu'il révèle la tension sur laquelle se construit l'identité féminine au sein de la maison close : perçue comme simple objet à plaisir, la femme est soumise à un phénomène de réification par le masculin; désireuse d'exister par elle-même, elle lutte pour marquer son individualité ainsi que son irréductibilité au désir de l'homme. Le toucher va donc tantôt symboliser la valeur « marchande » et «transactionnelle » du corps féminin soumis aux injonctions du masculin, tantôt la force du lien affectif qui unit ces femmes entre elles et leur offre un espace au sein duquel elles peuvent laisser libre cours à leurs émotions.

6 Le toucher qui structure les relations masculin/féminin constitue un acte ritualisé, gouverné par la dichotomie objet/sujet, où la présence féminine est réduite à son habileté à satisfaire les fantasmes masculins ${ }^{4}$. Le corps féminin apparaît alors domestiqué et inféodé aux désirs de l'homme, relayé en cela par un régime visuel de la contrainte qui « enferme » les personnages féminins, souvent immobiles, au sein du plan, les privant de leur liberté de mouvement en les obligeant à obéir aux directives du client. Au contrôle physique du corps répond le diktat du paraître, la peau de la femme s'inscrivant au sein d'une représentation extrêmement genrée où la surface a valeur expressive ${ }^{5}$. Deux scènes sont particulièrement éclairantes sur ce point. Chacune d'elles se fond sur une compréhension similaire du toucher comme acte hiérarchisant entre les sexes, signifiant le déni de l'altérité et confinant le féminin à sa dimension charnelle. Chaque scène représente une prostituée en compagnie d'un client, ce dernier leur demandant de jouer un rôle en se déguisant, l'une en geisha, l'autre en un automate grandeur nature. 


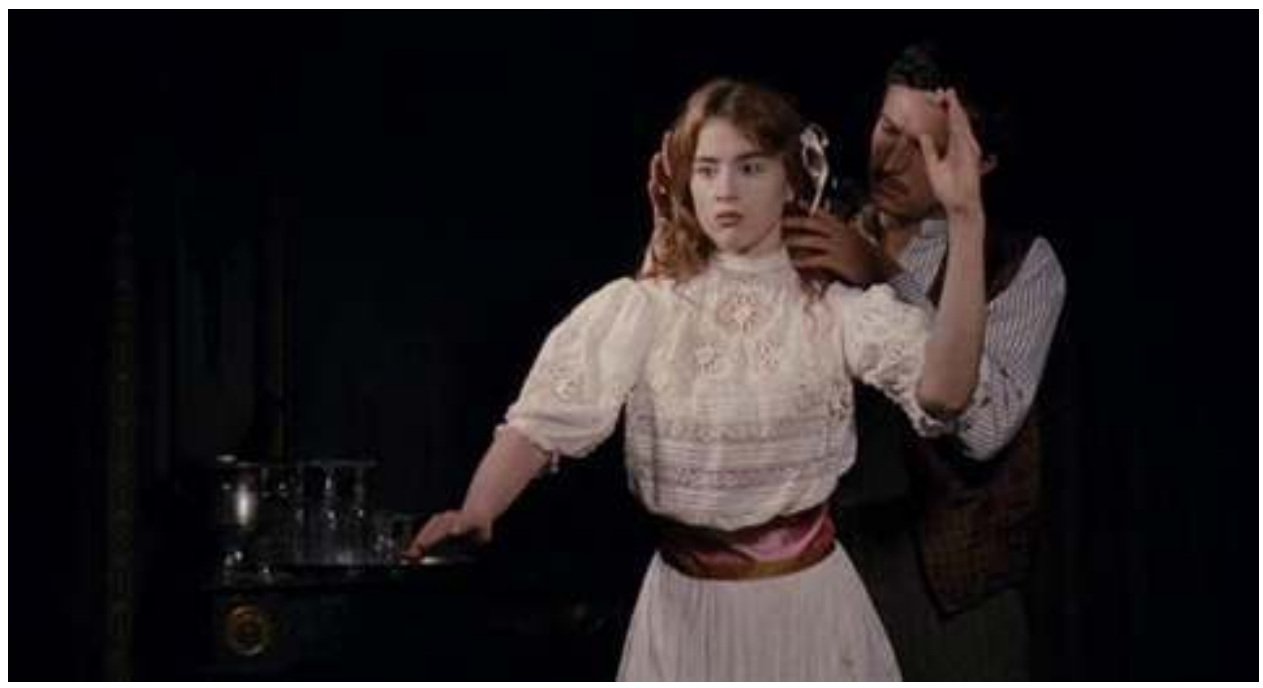

Figure 1 : Toucher masculin et réification du corps féminin

7 La beauté des costumes, le soin apporté aux coiffures ainsi qu'au maquillage concourent à faire de la femme un pur objet de désir prompt à satisfaire les plaisirs masculins.

8 Le toucher s'inscrit alors comme un acte symbolique de la domination masculine, puisque nous voyons les mains des clients se poser sur les corps des femmes, soit pour les dévêtir, soit pour y imprimer leur marque en leur indiquant avec minutie quelles poses prendre. Il $\mathrm{y}$ a ici corruption du féminin par le toucher, dans la mesure où :

Le corps est directement corrompu lorsque le corrupteur s'en empare comme de la chair inerte, sans esprit. Et le corps colonisé sous pression, se sent pris pour une chose, un objet, une image presque vivante. ${ }^{6}$

$\mathrm{Du}$ contact entre les corps émerge alors un sentiment d'annihilation de l'individualité féminine au profit d'une sur-expressivité de l'extériorité. Sentiment renforcé par le jeu de rôle auquel se livrent les prostituées, forcées d'abandonner jusqu'à leur apparence physique pour endosser celle d'une autre. La peau devient ici une surface inscriptible dont s'empare le masculin en l'instrumentalisant afin qu'elle réponde à son désir. Le toucher s'approprie la chair comme si elle n'appartenait plus à la femme, cette dernière se trouvant infériorisée du fait de la " pratique du corps $»^{7}$ inhérente à sa profession.

La fétichisation du corps de la femme, et son appropriation par le masculin, sont redoublées au niveau extra-diégétique par une mise en scène fondée sur un régime visuel optique $^{8}$. Le spectateur est tenu à distance des images, ces dernières suscitant un regard détaché, capable d'embrasser la représentation dans un mouvement unique de contemplation et de possession. La froideur des tonalités qui dominent chacune des scènes - le noir du décor contraste fortement avec la blancheur de la peau des femmes associée aux soins apportés aux costumes et aux poses figées des jeunes femmes, contribuent à faire de ces plans de véritables tableaux vivants. Le regard des spectateurs ne connait alors aucune entrave et glisse sur les corps afin de se les approprier dans la matérialité de leur présence physique. En outre, le caractère spéculaire de ces scènes (le client tient le rôle du metteur en scène et la prostituée celui de l'actrice qu'il dirige) vient souligner leur caractère extrêmement construit. Le corps féminin ne fait sens qu'à travers son "savoir-paraître ». Au geste intrusif du masculin répond ainsi une mise en image archétypale du corps féminin, source de satisfaction visuelle qui crée un nivellement du sens. Le corps n'est plus compris qu'à travers sa dimension charnelle, cette dernière 
effaçant toute intériorité afin de nous sensibiliser au fait que « [1]a chair est le corps mais elle est séparée du sujet, elle est extériorisée, lubrifiée, cirée, elle sert d'apparence, de faire valoir à l'identité. $»^{9}$

\section{Toucher et subjectivité féminine}

11 À la concrétude du corps féminin, et sa soumission au masculin, s'oppose l'ineffable du ressenti, et c'est en faisant également de la caresse la marque du lien qui unit les femmes de la maison close que le film fait du toucher un acte aux ramifications complexes. Il signifie autant la face sombre de leur servitude que celle, plus lumineuse, de leur entraide et de leur instinct de résilience face aux drames de la vie.

12 À ce titre, la scène où nous voyons les jeunes femmes danser entre elles et se consoler après qu'une des leurs soit morte de la syphilis offre une nouvelle illustration de la force affective et structurante qui émerge de la rencontre des corps.

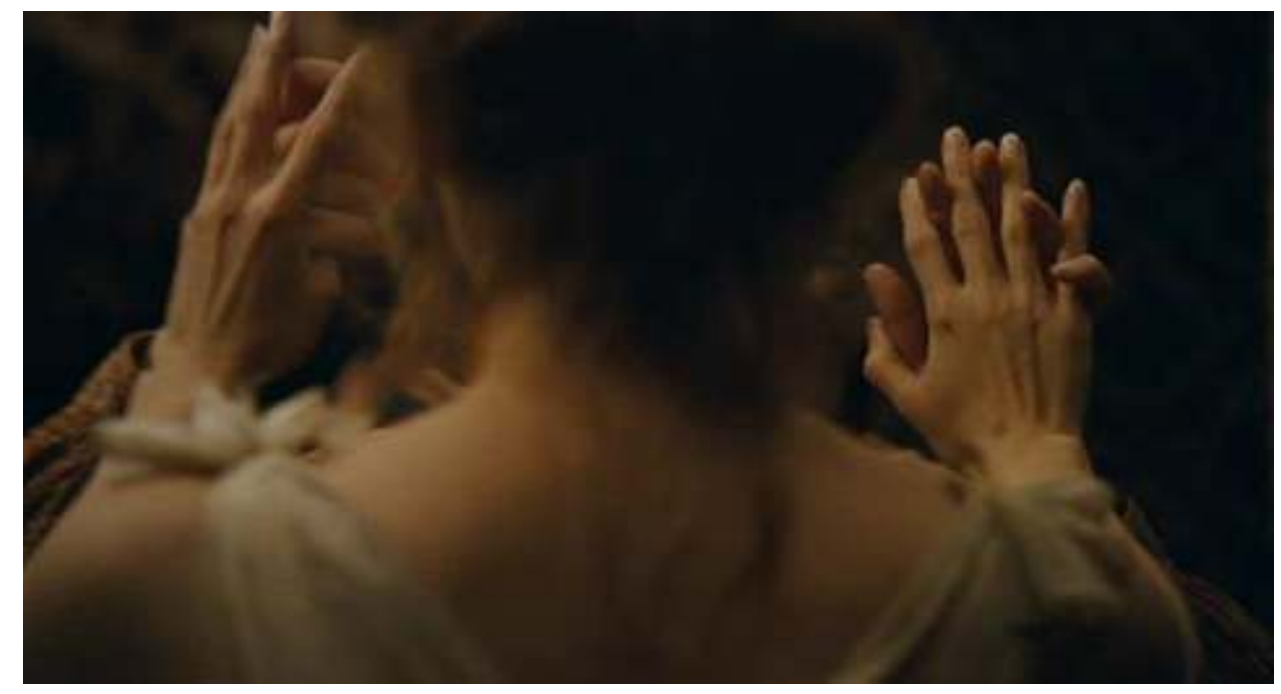

Figure 2 : Toucher féminin et pouvoirs de l'affect

Cette scène se présente comme l'antithèse de celles analysées précédemment, tant sur la symbolique du toucher qui s'y met en place que sur la nature du rapport entre les spectateurs et les images.

Si les scènes précédentes fonctionnaient sur le mode de la répression de l'individualité et de la contrainte des corps, celle-ci est au contraire travaillée par la volonté d'offrir un espace propre à l'expression de la subjectivité féminine, en permettant aux affects de circuler librement au sein du groupe et de venir façonner la représentation. La présence masculine ayant été complètement évacuée, le toucher n'a plus ici cette valeur hiérarchisante, en ce qu'il ne sert plus à établir une distinction sujet/objet, dominé/ dominant. Il porte au contraire la marque de la singularité de chaque femme qui trouve à s'exprimer dans la pluralité du groupe. Au statisme des femmes dans les scènes précédentes, lequel correspondait à l'aval du masculin sur le féminin, vient ici s'opposer la liberté de mouvement et la fluidité des corps. Les reconfigurations permanentes auxquelles ils donnent lieu symbolisent la libre circulation des émotions et la possibilité de faire l'expérience de soi en-dehors de toute contrainte. La tactilité joue un rôle prépondérant, tant au niveau diégétique qu'au niveau extra-diégétique, puisque le 
changement que nous notons dans la symbolique du toucher est intrinsèquement lié à la manière dont cette scène nous est communiquée par le film.

À la position de simple voyant que nous occupions dans les scènes mentionnées précédemment se substitue l'investissement émotionnel et sensoriel transmis ici par les images. La lumière douce et chaude qui vient éclairer les corps, les gros plans sur les mains des femmes qui s'effleurent, sur les corps qui se serrent ou sur les nuques délicates sont autant d'éléments qui éveillent les sens du spectateur et l'invitent à s'approcher au plus près des images pour en saisir toute la dimension affective. La mise en scène cherche à attirer le regard du spectateur dans la matérialité de l'image afin de faire naître des émotions qui dépassent le cadre de l'intellection pour en appeler à un savoir sensitif du corps.

Ce phénomène s'accompagne d'une réévaluation de l'expérience cinématographique, puisqu'il ne nous faut plus penser l'écran de projection comme une surface sans aspérités, mais davantage comme une membrane nous permettant d'établir un contact intime et sensoriel avec la matière foisonnante des images. ${ }^{10}$ Les spectateurs et le film communiquent alors sur le mode de la réciprocité, puisque nous touchons le film autant que nous sommes touchés par lui, et ce dans un phénomène d'imprégnation mutuelle. ${ }^{11}$ Ramenée à la scène analysée, une telle approche de la relation film/spectateur nous permet d'aborder la fragilité, la tristesse, ainsi que l'élan vital qui habitent ces femmes comme autant d'émotions appartenant au domaine du non-verbal, et naissant du contact des corps à l'écran. Cette force émotionnelle dépasse les corps eux-mêmes et l'univers diégétique pour s'inscrire dans la matière même de l'image, puisque c'est à travers elle que nous sommes appelés à entrer en contact avec le film et à ressentir en nous la complexité des sentiments qui parcourent ces corps et donnent leur chair aux images.

Comme le souligne Jennifer Barker dans son livre The Tactile Eye :

L'amour, la perte, la nostalgie et la joie sont des émotions qui trouvent essentiellement à s'exprimer par des moyens tactiles, non seulement entre les personnages, mais également, et de manière plus intime, entre le film et le spectateur. ${ }^{12}$

Le concept de «tactilité » tel qu'il nous faut le comprendre à présent ne correspond plus seulement au contact physique concret qui se noue entre les personnages au sein du récit, mais comme ressortissant du type de contact généré par l'image en direction du spectateur. Le mode optique, qui prévalait jusqu'alors dans notre compréhension de la représentation, est remplacé par le mode haptique «[...] où dominent la matérialité de l'image et des sons [où] la figure humaine apparaît comme un élément changeant, en mutation, au milieu d'un univers fait de lumière, de couleurs, de textures, de lignes et de formes $»^{13}$. Nous passons ainsi d'un regard détaché et tout-puissant à un regard qui accepte de lâcher prise et de perdre le contrôle face aux images dont le sens est en perpétuelle évolution.

19 En franchissant symboliquement la surface de l'écran de projection, le film nous permet d'être au plus près de l'intimité des femmes de la maison close et d'approcher la complexité de leur condition, où l'insouciance et la légèreté - factices - qui président à leur métier côtoient la solitude, la mort et la peur du lendemain. En nous invitant « [...] à ressentir plutôt qu'à voir le film, à entrer en contact par le biais de la peau $»^{14}$, les images délestent le regard de toute forme de jugement moral pour en arriver à lui faire partager l'espace émotionnel de ces femmes. Il devient alors possible pour ces dernières de se 
réapproprier leur corps et leur identité, puisque l'espace de l'écran n'est plus une menace à leur intégrité, mais au contraire le lieu propre à l'expression du corps vécu subjectif.

\section{Un corps à corps entre le film et le spectateur}

20 Si nous nous sommes jusqu'à présent intéressés au toucher comme manifestation affective naissant d'un contact à la surface de la peau, nous souhaitons à présent observer comment la rencontre entre les corps peut s'effectuer sur un mode plus menaçant, lorsqu'elle ne se limite plus à l'enveloppe extérieure et que la peau en vient à être meurtrie. Cette forme particulière de toucher constitue une force perturbatrice et va infléchir la chronologie de la narration, cette dernière se trouvant bouleversée par l'expérience traumatique de l'une des prostituées, Madeleine, dont le visage a été profondément tailladé par l'un des clients. Cet événement apparaît comme la pierre d'achoppement du récit, dont la forme concentrique revient à intervalles réguliers buter sur l'horreur et l'indicible d'un tel acte. En effet, la résurgence de cette scène n'est pas motivée par une nécessité narrative (le spectateur n'a pas besoin de ces retours en arrière pour comprendre ce qui est arrivé à la jeune femme) mais bien par la puissance émotionnelle qu'elle contient. La force affective qui émerge de la violence de ce contact physique et les séquelles - physiques autant que psychologiques - que cela va entraîner chez Madeleine, dépassent le cadre de la représentation pour faire surface en différents points du récit. La scène traumatique est prise dans une boucle temporelle au sein de laquelle elle est appelée à se rejouer à l'infini.

21 Là encore, la peur et l'effroi qui émergent de la violence faite au corps féminin ne sont pas des données qui existent a priori, mais bien le produit de la rencontre des corps et de leurs attitudes l'un envers l'autre. C'est dans la tension générée par le mouvement de la main, munie d'un couteau, sur le corps de Madeleine, face au statisme de la jeune femme attachée au lit, qu'émerge le sentiment d'horreur lié au toucher. Ce sentiment est communiqué aux spectateurs par le biais d'une mise en scène qui cherche à mobiliser les fonctions internes et musculaires du corps, afin de pouvoir véhiculer par l'image la subjectivité de l'expérience vécue par le personnage féminin.

22 À l'inverse de la scène analysée précédemment, où le film entrait en contact avec le spectateur sur le mode délicat de la caresse, les images font naître ici des sensations plus profondes et viscérales. Ces manifestations sont le fait d'un travail conjoint sur le son, la lumière et l'échelle des plans dont le but est de faire transparaître à l'image le dénuement physique et psychologique dans lequel se trouve la jeune femme. La scène fonctionne sur une série de gros plans sur le visage et le buste de Madeleine, tandis que nous ne voyons de l'homme que sa main, qui promène la lame du couteau sur la poitrine de la jeune femme, s'arrêtant sur la pointe d'un sein pour finir par glisser dans sa bouche. 


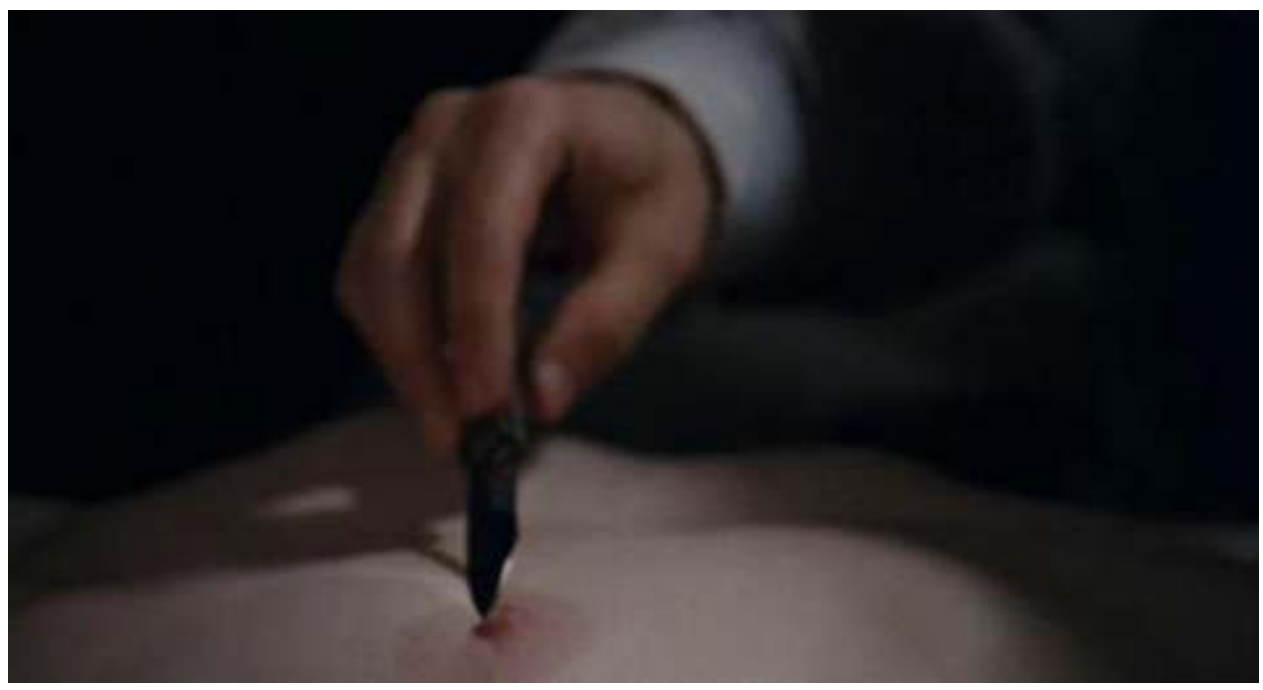

Figure 3 : L'horreur du toucher dont elle fait l'expérience à travers la terreur qui se noue entre elle et son client, et qui se propage dans sa chair, viennent également "s'imprimer» chez le spectateur. Le film exprime cet enfermement physique et psychique dans l'espace de la terreur en nous attirant spatialement et viscéralement vers lui, principalement par le biais des gros plans dont l'effet centripète tend à nier le hors champ afin de focaliser l'attention sur l'action au sein du cadre. La tension et la contraction musculaires que nous ressentons, l'accélération du rythme cardiaque, le souffle qui devient court sont autant de manifestations internes de notre corps qui régissent notre rapport aux images et nous révèlent dans l'inorganisé de notre intériorité, ainsi que dans notre intimité la plus profonde.

26 Le toucher, dans le film de Bertrand Bonello, apparait comme un geste porteur d'une grande richesse sémantique, et dont l'étude nous permet de mettre en avant les qualités texturelles du film. En nous révélant les différentes implications qu'ont les diverses formes de toucher dans notre compréhension du féminin, le film vient aussi nous toucher en 
déployant un univers visuel qui va susciter en nous une variété de réponses somatiques et nous faire entrer en contact avec la chair des images. En nous indiquant que le toucher peut se faire caresse aussi bien que blessure, le film nous sensibilise à la peau en tant que surface signifiante, dans ce qu'elle masque autant que dans ce qu'elle révèle. Ce phénomène se joue aussi bien sur le plan diégétique que sur le plan extra-diégétique, en ce que le film nous permet également de faire l'expérience de notre propre corporalité en remettant perpétuellement en question notre position face aux images. Qu'il nous tienne à distance, nous amène avec douceur près des corps ou nous presse brutalement contre eux, le film cherche avant tout à faire des spectateurs une composante active du récit, ne leur assignant pas une place définie, mais appelant au contraire à éprouver dans et sur leur corps les fluctuations internes du « corps-film ».

\section{NOTES}

1. Laure Adler, La Vie quotidienne dans les maisons closes, Paris, Hachette, 1990, p. 68.

2. Sarah Ahmed, The Cultural Politics of Emotion, New York, Routledge, 2004, p. 9.

3. Ibid., p. 10.

4. S'il a par la suite donné lieu à certaines critiques, l'article fondateur de Laura Mulvey "Visual Pleasure and Narrative Cinema" a permis de mettre à jour la nature extrêmement genrée de la représentation dans le cinéma traditionnel. Dans son article, Mulvey considérait l'œil de la caméra comme le prolongement du regard masculin, en ce que le corps féminin était avant tout considéré comme objet propre à répondre aux désirs du masculin, la mise en scène perpétuant ainsi les codes du patriarcat.

5. Le travail effectué dans ces scènes sur la pâleur et le lisse de la peau des jeunes femmes renvoie aux oppositions qui ont longtemps structuré la compréhension du corps masculin et du corps féminin. Le premier étant considéré comme chaud, mais aussi plus sec et donc plus résistant que le corps féminin, ce dernier se définissant par sa douceur, sa souplesse et sa malléabilité. Voir Constance Classen, The Deepest Sense. A cultural History of Touch, Urbana, University of Illinois Press, 2012, pp. 71-72.

6. Steven Bernas, «Corrompre la chair », La Chair à l'image, Steven Bernas et Jamil Dakhlia (eds.), Paris, L'Harmattan, 2006, p. 79-80.

7. Ibidem.

8. Nous allons revenir plus longuement sur la distinction entre vision optique et vision haptique au cours de notre étude. Pour une meilleure lisibilité du texte, nous définirons ici la vision optique comme correspondant à la relation qui se met en place entre les spectateurs et la représentation lorsque cette dernière se fonde sur le concept de vraisemblance. Le respect de la perspective permet un rapport non problématique entre le spectateur et la fiction, cette dernière ayant pour but de conforter la toute-puissance du regard de celle ou celui qui perçoit.

9. Steven Bernas, « Corrompre la chair », op. cit., p. 77.

10. Voir les ouvrages suivants : Jennifer M. Barker, The Tactile Eye. Touch and the Cinematic Experience, Berkeley, University of California Press, 2009 ; Martine Beugnet, Cinema and Sensation. French Film and the Art of Transgression, Edinburgh, Edinburgh University Press, 2007 ; Laura U. Marks, Touch: Sensuous Theory and Multisensory Media, Minneapolis, University of Minnesota Press, 
2002 ; Vivian Sobchack, Carnal Thoughts: Embodiment and Moving Image Culture, Berkeley, University of California Press, 2004.

11. Notre compréhension du rapport de réciprocité qui s'instaure entre le film et le spectateur se fonde sur les théories de Merleau-Ponty selon lesquelles l'humain fait l'expérience du monde sensible sur la base de la réciprocité et de la réversibilité.

12. Jennifer M. Barker, The Tactile Eye: Touch and the Cinematic Experience, op. cit., p. 1. L'auteur traduit.

13. Martine Beugnet, Cinema and Sensation. French Film and the Art of Transgression, Edinburgh, Edinburgh University Press, 2007, p. 60. L'auteur traduit.

14. Jennifer M. Barker, op. cit., p. 23. L'auteur traduit.

15. Sara Ahmed, The Cultural Politics of Emotion, op. cit., p. 6. L'auteur traduit.

\section{RÉSUMÉS}

Cet article se propose d'étudier le film de Bertrand Bonello, L'Apollonide (2011) en s'intéressant aux différentes formes que recouvre le toucher au sein du film. Nous constaterons que c'est à travers la symbolique du toucher que se dessine la complexité de la figure féminine mise en place par le récit. En choisissant de nous présenter le quotidien d'une maison close à la fin du XIXème siècle, le film dépasse le simple cadre illustratif afin de nous amener au plus près du vécu subjectif de ces femmes. Nous observerons alors que le toucher n'est pas une donnée propre à l'univers diégétique, en ce qu'il va également définir le contact qui se noue entre le spectateur et les images, ces dernières appelant divers types d'investissements physiques et émotionnels, faisant tour à tour du spectateur un simple voyant ou un participant actif du récit.

Understanding Through the Senses: For a Tactile Approach of L'Apollonide

This article offers to analyze the significance of touch in Bertrand Bonello's L'Apollonide: Souvenirs de la maison close (2011). I will observe how touch operates as a structuring device within the narrative, as it serves to evidence the complex status of the women within the house of tolerance. I will show that touch can embody the women's subjection to masculine desires, but also their wish to break free from the constraints of their profession and to express their inner feelings. I will highlight how the mise-en-scène works to elicit various corporeal involvments on the spectator's behalf that mimic this duality at heart of the representation. The spectator is either asked to remain at a distance from the image and to consider the women as pure objects of desire, or he is invited to emerge himself within the fabric of the image to experience the women's subjectivity and emotions.

\section{AUTEUR}

\section{ROMAIN CHAREYRON}

A obtenu son doctorat à l'université de l'Alberta (Edmonton, Canada) en 2010. Sa recherche porte sur l'influence de l'horreur et de la pornographie dans le cinéma français contemporain. Ses travaux actuels s'intéressent au concept de « monstruosité » et à ses implications esthétiques 
dans le cinéma français des années 2000. Il enseigne la littérature et la culture françaises dans le département de français et d'italien à l'université du Kansas. 